\title{
Corela
}

Cognition, représentation, langage

HS-28 | 2019

Les affordances langagières : textualité numérique, matérialité discursive

\section{Entre perception et praxis : à quoi la notion d'affordance en discours peut-elle bien pourvoir?}

\section{Laurent Fauré}

\section{CpenEdition}

Journals

Édition électronique

URL : http://journals.openedition.org/corela/8434

DOI : $10.4000 /$ corela.8434

ISSN : 1638-573X

Éditeur

Cercle linguistique du Centre et de l'Ouest - CerLICO

Référence électronique

Laurent Fauré, « Entre perception et praxis : à quoi la notion d'affordance en discours peut-elle bien pourvoir? », Corela [En ligne], HS-28 | 2019, mis en ligne le 11 septembre 2019, consulté le 10 décembre 2020. URL : http://journals.openedition.org/corela/8434 ; DOI : https://doi.org/10.4000/ corela.8434

Ce document a été généré automatiquement le 10 décembre 2020.

\section{(i) 80

Corela - cognition, représentation, langage est mis à disposition selon les termes de la licence Creative Commons Attribution - Pas d'Utilisation Commerciale - Partage dans les Mêmes Conditions 4.0 International. 


\title{
Entre perception et praxis : à quoi la notion d'affordance en discours peut-elle bien pourvoir?
}

\author{
Laurent Fauré
}

\section{Introduction}

\begin{abstract}
$\mathrm{Au}$ volant d'un petit camion de chantier, un conducteur manœuvre prestement entre les obstacles, les façades et les piétons; tout à coup, mettant le véhicule au point mort et utilisant son inertie, il se rapproche délicatement et silencieusement d'un passant dont, avant de s'arrêter, du rétroviseur, il touche précautionneusement l'épaule, de dos. Surprise de l'intéressé, qui étouffe une vocalisation interjective et se retourne pour se plaindre : et le piéton de découvrir, à travers le pare-brise qui lui fait face, le visage souriant de l'ami avec lequel il engage de belle humeur la conversation, appréciant la plaisanterie...
\end{abstract}

1 L'anecdote ci-dessus, notée sur le vif il y a quelques années dans une petite rue du centre de Florence permet de questionner le rapport entre inventivité et invites perceptuelles qui nous font tirer profit de nos environnements dans nos pratiques situées quotidiennes. Cette perspective convoque la complexité des relations entre le corps phénoménologique et ses prolongements techniques, nos facultés d'adaptation parmi les attentes sociales, le recours, ou pas, au langage... À ce titre, l'affordance s'avère un concept commode. Ses facilités le rendent certes perméable à des critiques de divers ordres mais il présente d'indéniables avantages pris en un sens englobant, dynamique et émergent. C'est du reste ainsi que le conçoivent l'ensemble des contributions de la présente livraison de Corela. La démarche du psychologue Gibson qui a modelé, dans la lignée de Lewin et de la psychologie de la forme, la notion d'invite perceptuelle, est surtout l'une des premières, contrairement aux approches causales et inférentielles traditionnelles, à s'intéresser précisément à notre rapport perceptuel aux objets et surtout à ce que nous faisons du dit rapport comme sujets. Aussi la proposition, soutenue notamment par Paveau (2013) de 
transférer cette notion psychologique à l'observation des données langagières semble d'autant plus bienvenue qu'elle porte sur le rapport à la signification, ou plutôt, pour se conformer à la conception de Gibson, à l'activité de signifiance. Le présent travail se propose de revenir d'abord sur diverses (re)lectures successives de l'affordance, dans le cadre de l'approche écologique (1.) ou dans une perspective plus critique (2.), qui ont toutes envisagé le rapport de la notion au réel, à l'action et à l'environnement, préparant ainsi (3.) les réflexions propres à la dimension proprement linguistique et discursive. Un dernier volet (4.) sera consacré à une étude de cas qui permettra de mettre la complexité des formats d'affordance au sein d'un centre de vidéosurveillance urbaine. Dans une optique praxéologique et interactionnelle qui puise à diverses sources (ethnométhodologie, praxématique, anthropologie linguistique et approche énactiviste) et croise assidûment la route d'autres linguistes, notamment sémioticiens et ceux du réseau des linguistiques TDI, au sens initié par M.-A. Paveau, j'envisagerai plus particulièrement en quoi l'activité perceptuelle et interprétative emprunte aux praxis, dans des cours d'action supportés langagièrement.

\section{L’approche écologique}

\subsection{Cadrage théorique initial}

2 La première version de l'affordance selon Gibson emprunte aux formulations initiales de Werner $(1926)^{1}$ et de Lewin ${ }^{2}$ (1936) et à la tradition de la psychologie de la forme : elle convoque une lecture physiologique directe, sans tâche complexe de traitement de l'information, selon la même procédure que celle valant pour les émotions. Quant au vecteur de l'opération, Gibson affirme que les invariances (lumière ambiante / optique) spécifient les propriétés environnementales directes. C'est à elles, qui sont toujours là, indépendamment des sujets, que l'on va " prêter attention ». Aussi, les affordances, dans la première version de Gibson, sont-elles radicalement indépendantes du sujet percevant (c'est la boîte à lettres physique, en tant que telle, et non phénoménale qui procure la possibilité d'y jeter une lettre); elles seront saisies sous les espèces de l'émergence, ultérieurement chez ses disciples (Turvey \& alii, 1981). L'affordance peut donc se définir comme le principe offrant à un sujet qui en a la conscience toutes les actions potentielles qu'il est en mesure d'effectuer sur un objet donné. Si propriété intuitive il y a, elle renvoie à l'invite qui est faite au dit sujet par ledit objet à en faire l'usage le plus pertinent.

\subsection{La poule et l'œuf}

On peut estimer que la conception de l'affordance a quelque peu évolué, y compris au sein de la pensée de Gibson, allant d'une formulation à vocation relativement déterministe pour accéder à une approche plus interactive. Pour rester dans le même registre de simplification sommaire, on peut considérer que la première version, encore très proche des gestaltistes, correspond à celle qui consiste à obéir à la forme, la structure et la consistance de la roche qui s'imposent ainsi à la sculpture (cf. Figure 1) ; la conception relue propose en revanche de voir dans le geste du sculpteur l'intelligence de la matière qu'il façonne en tenant compte, certes, des contraintes, mais en y adjoignant sa vision. L'expérience du sujet (qu'il s'agisse ou non de l'expression de sa volonté) épouse les contraintes de la matière, en la transformant en offre, en quelque sorte. 




Figure 1 : Formulation initiale (déterministe) de l'affordance

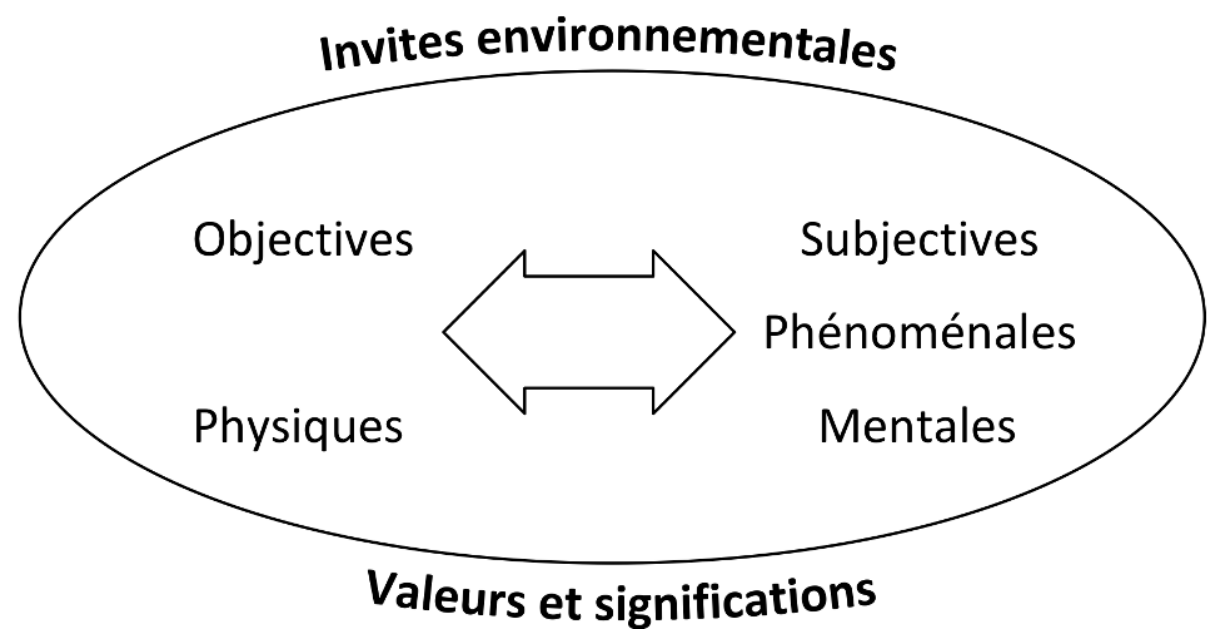

Figure 2 : Seconde version de l'invite (dialectique)

4 Une invite est ainsi autant façonnée par l'environnement que par le comportement (Figure 2). Or, comme celui-ci consiste in fine à interpréter le premier on est mené à constater que la place de l'agent (l'organisme) devient décisive, ce qui renvoie à une vision utilitariste de l'affordance. Toute la difficulté devient dès lors l'établissement du rapport qu'entretient cette dernière avec l'intentionnalité, liée au caractère finalisé de l'interprétation. Toutefois, Gibson met en garde lui-même contre ce retour à une conception classique : c'est, dit-il d'un «objet écologique à valeur riche » que procède l'activité perceptuelle qui va faire sens. Et de réfuter toute lecture d'une activité de perception donnant une interprétation à un objet dont la valeur a priori serait indécidable. Du reste, le même passage donne aussi l'occasion au psychologue de se départir explicitement des théories de la valeur et du sens ${ }^{3}$. On peut ainsi comprendre que les données de l'environnement sont fortes de leurs propriétés qui constituent ellesmêmes des invites lesquelles guident le sujet perceptuel dans sa prise en considération des dites données. Il s'agit pour lui de saisir, d'appréhender des informations qui s'offrent à sa perception et dont il va modeler le profit qu'il peut en retirer. Les objets ne sont ainsi ni vides non neutres mais exhibent plus ou moins leurs propriétés dont les organismes peuvent tirer parti. D'un autre côté, cela suppose que ces derniers assument une vision des choses liée à leur propre expérience - et c'est tout l'enjeu de l'apport de Gibson -, leur nature spécifique les y amenant.

5 Il y a toutefois comme un mouvement de repentir permanent dans cette conception dont on mesure bien qu'elle préfigure précisément les théories de l'émergence. Ce relatif 
flottement - c'est un peu la bouteille à l'encre -, pour novateur qu'il ait été, n'a pas manqué de susciter plusieurs séries de critiques. Pour en rappeler quelques unes, on commencera par celles qui relèvent du champ de la cognition.

\subsection{Le correctif de Norman}

6 Un premier assouplissement théorique a été apporté par D. Norman, un concepteur en design qui a su popularisé l'affordance et en a d'abord revendiqué pleinement la notion, avant d'en proposer un adoucissement. Il reprend tout d'abord à son compte le cadre définitoire de l'approche écologique :

«Les affordances fournissent des indices forts à l'exploitation des choses. Les plaques sont faites pour pousser. Les boutons sont destinés à tourner. Les fentes sont pour y insérer des choses. Les balles sont destinées à être lancées ou rebondir. Lorsque les affordances sont avantageuses, l'utilisateur sait ce qu'il faut faire en regardant : aucune image, étiquette ou instruction n'est requise. $»^{4}$ (Norman, [1988]2002:9)

7 Mais l'instantanéité, non construite et non médiée de l'invite chez le sujet ne laisse pas de renvoyer à une vision quelque peu impressionniste et circulaire de l'instinct. Aussi, l'auteur tient-il à préciser sa propre acception du terme :

"Je pense que les affordances résultent de la représentation mentale des choses, qui repose sur nos savoir et expérience passés appliqués à notre perception des choses sur nous. $»^{5}$ (Norman, [1988]2002 : 219)

Cette relecture de l'affordance qui donnerait matière à apprentissage en relativisant le caractère direct de la perception dans l'approche écologique, a fait précisément le succès de la notion. Il faut reconnaître qu'elle est davantage aisée à agréer car plus classique que la version plus radicale de son promoteur initial. De fait, contrairement à Gibson et le revendiquant tout en atténuant la portée de la distinction, Norman (2002:119), participe de la sorte aux paradigmes de la partition entre l'activité cognitive (propre à l'esprit) et celle du corps et de l'environnement. Cette conception binaire de la cognition (dite aussi "en sandwich») renoue avec un dualisme traditionnel que Gibson avait lui-même dépassé. Sur un point essentiel toutefois, celui de la différence entre affordances perçues et réelles, le designer fera amende honorable (Norman, 1999: 39), regrettant la circulation illicite à ses yeux de la confusion entre les deux instances, induite par son propre travail, en particulier au sein de sa sphère professionnelle (et des créateurs d'interface sur écran). Il sera ainsi amené à rectifier ses formulations précédentes dans des éditions ultérieures de The Design of Everyday Things. Pour lui, en réalité, les «affordances » technologiques n'en sont pas toujours ou jouent un rôle subalterne dans nombre de dispositifs qui relèvent plutôt de "conventions culturelles ». On le voit: le dossier se complique d'un usage intempestif d'une notion (trop) commode à manipuler pour ne pas... pourvoir ellemême à des raccourcis, lesquels ne résolvent pas le problème de la teneur perceptuelle des invites.

9 Un premier bilan permet de clarifier : selon Gibson, l'affordance est indépendante du sujet et de ses aptitudes et $a$ fortiori de sa culture, de ses connaissances, de son expérience, elle existe, en soi comme une possibilité - quand elle permise physiologiquement à un organisme - de faire quelque chose avec un objet de l'environnement et ne nécessite pas de médiation cognitive. Pour Norman, elle est reliée à la capacité perceptuelle du sujet et 
peut être corrélée à sa culture, son expérience, son savoir d'arrière-plan de sorte qu'il peut percevoir comme affordance des propriétés qui ne sont inhérentes à l'objet.

\section{Relectures et controverses}

\subsection{De la philosophie analytique à la critique ethnométhodologique}

10 Le correctif de Norman, travaillé par un regard professionnel tourné vers les usages et non, comme il le dit lui-même, vers la teneur des invites qui seraient réellement conssubstantielles aux objets, suscite un important distinguo: entre affordances réelles, naturellement données en quelque sorte et affordances perçues, se jouent tout l'édifice culturel et le faisceau, décisif, aux yeux de l'auteur, des « conventions » y afférant. Cette précision renvoie directement aux pages fameuses de Wittgenstein (1961[1953] : 326) sur la perception visuelle: "nous voyons [un objet/son illustration] comme nous l'interprétons "; et le philosophe de convoquer le croquis du lapin-canard, inspiré du psychologue Jastrow, que l'on peut voir tantôt comme le volatile tantôt comme le rongeur aux longues oreilles, image sur laquelle il appuie sa « distinction entre la "vue constante" d'un aspect et sa "naissance" ». En d'autres termes, on peut continuer à voir un lapin mais, dès que l'on est mis sur la piste perceptuelle d'un canard cela implique que les mêmes traits formels sont reconfigurés interprétativement comme propriétés sémiotiques nouvelles. Selon Wittgenstein, on communique sa perception puis, si on est amené à changer d'impression visuelle, on enregistre ce changement d'état :

11 "si vous regardez un objet, vous n'avez pas besoin d'y penser; mais dès que vous éprouvez l'expérience visuelle, exprimée par l'exclamation ${ }^{6}$ [qui, à la différence de l'annonce, s'arrache à nous], vous pensez également à ce que vous voyez » (Ibid. : 329)' ${ }^{7}$. Si l'expression de la surprise face à la nouvelle forme émergente met en évidence une transition, l'existence a contrario d'attentes d'arrière-plan qui valent pour autant d'allants-de-soi, conforterait plutôt l'approche gibsonienne d'une affordance sans médiation, même si elle repose - et cela apporte de l'eau au moulin de la version de Norman - sur des prérequis conceptuels : des précatégorisations. L'invite relèverait ainsi d'un soubassement relevant de la cognition distribuée (en relation avec les attentes sociales), au sens contemporain, depuis Hutchins. Il ne me semble pas que cette reconsidération remette en question la perception directe revendiquée par Gibson. Ce dernier visait en effet un dépassement de l'explication de cette dernière selon les psychologues de la forme par la tension vectorisée entre objet et ego phénoménaux, qu'il jugeait inutilement sophistiquée et régressive (Gibson, 2014 [1979]: 226-229). Une précision du psychologue vient toutefois compliquer le dossier :

«Le concept d'invite est dérivé de ces concepts de valence, d'invitation et d'exigence [issus des gestaltistes], mais avec une différence cruciale : l'invite de quelque chose ne change pas en fonction des changements des besoins de l'observateur. L'observateur peut percevoir ou ne pas percevoir l'invite, y être attentif en fonction de ses besoins, mais l'invite, étant invariante, est toujours présente et susceptible d'être perçue » (Gibson 2014 [1979] : 227).

12 Cette invariance de l'invite revient à lire l'image du canard ou du lapin, comme commandée par des propriétés antécédentes à toute perception, en soi. Or, la syntaxe wittgensteinienne du voir comme contrevient directement à cette assomption puisqu'elle implique apparemment à son tour une médiation conceptuelle et construite, voire 
intentionnelle. Aussi, dans leur charge contre la psychologie écologique, Fodor et Pylyshin (1981: 188 sqq), n'ont pas manqué de relever ce point du raisonnement logique, en dégageant une composante inférentielle de la perception : voir un objet comme tel supposerait d'en retirer une indication qui revient à en donner une définition, guidée par le besoin que nous avons $\mathrm{du}$ dit objet. Toutefois, dans une perspective ethnométhodologique, Coulter et Parsons (2002[1990] : 221), de leur côté, et à la suite de Shanker, critiquent à leur tour cette conception inférentielle (réductionniste) comme « illusion de la représentation interne » : comme le cerveau ne reconnait rien, il n'est pas guidé par un jeu d'informations dont il ne sait rien mais supporte le processus mental de décryptage du réel, via un niveau conceptuel dont la maîtrise est corrélée à l'expérience pratique et partagée. Ce que nous percevons est analysé en termes sensibles et non le contraire... La carence de la psychologie écologique qui a su, de façon novatrice, articuler mouvement et perception, se situerait plutôt du côté de la faible part accordée à l'ensemble des activités humaines (Coulter et Parsons (2002[1990] : 224-225).

13 Cependant, reprenant le cas de la boîte aux lettres, Gibson susbsume l'opposition initialement portée par Koffka entre son caractère phénoménal et physique, pour y lire l'enregistrement d'un usage stabilisé socialement et culturellement « toute personne âgée de plus de six ans sait à quoi elle sert et où se trouve la plus proche » (Gibson, [1979] 2014 : 228). Ce qui intéresse le psychologue sous cette extension à des affordances perçues puis apprises, puis passées en perception directe c'est donc moins leur genèse que leur valeur d'usage, en quelque sorte.

Ajoutant de son côté la compétence des membres (à l'inclusion de sa dimension linguistique), à la congruence de l'activité, des perceptions, des mouvements, des faits et événements contextuels, l'ethnométhodologie a insisté sur l'interprétation séquentielle des environnements, sensibles aux actions progressives des participants. Plus globalement, le déplacement théorique considéré permet reconsidérer plusieurs sous plusieurs aspects la notion d'affordance.

15 La critique de Coulter (1999) se présente d'abord comme un plaidoyer en faveur de la psychologie gibsonienne, dont il partage plusieurs postulations. L'ethnométhodologue considère d'abord qu'on peut rendre compte de l'information sans se cantonner aux propriétés de l'objet mais d'un point de vue social et culturel, sur la base d'une véritable pensée de l'interrelation aux objets. Si l'approche s'avère en fait relativement classique, elle présente toutefois l'originalité de soulever explictement le questionnement suivant: "comment l'information délivrée par le monde est-elle transformée par le monde perçu ?». Précisément, pour Coulter et Sharrock (1998), l'information pose une difficulté définitoire à laquelle s'adjoint un problème de circularité, plus consistant: si, pour accéder à l'information, il faut en passer par l'affordance, celle-ci n'en doit pas moins être supportée (support, objet, symboles...) et pour détecter ladite information, il faut pouvoir la percevoir (voir, entendre, toucher...). Les auteurs n'envisagent sans doute pas pleinement, en l'occurrence, la spécificité du projet de Gibson, pour qui se manifestent d'autres fonctionnements informationnels que le rapport à la connaissance et au stimulus : cf. l'empreinte digitale, par exemple. Ils semblent oublier du reste la définition spécifique que donne Gibson de la notion d'information, bien différente en particulier de celle stabilisée au sens technique, depuis Shannon (et qui suppose le transfert par émission d'une source en direction d'un récepteur). On rappellera d'abord que le psychologue a progressivement mûri sa réflexion sur ce point, passant précisement du stimulus à l'information, saisie à la fois au sens commun et plus précisément comme 
énergie, en prise directe sur ce que suscite l'environnement. Pour Gibson, l'information n'est ainsi pas donnée à l'organisme. C'est, au contraire, ce dernier qui l'obtient en allant à elle, dans une démarche active. Il affirmera même que, d'un point de vue fonctionnel, animal et environnement «forment une paire irréprochable. Chacun implique l'autre » (Gibson, 1979 : 8).

Une notation importante de Coulter et Sharrock arrêtera le linguiste : celle du retour du langage nécessaire pour catégoriser un certain nombre de perceptions. Et les auteurs de prendre l'exemple de l'étamine: son concept permet de savoir quoi regarder (aspect qui renvoie lui-même aux bases phénoménologiques de la perspective des acteurs, selon Schutz): il entre assurément des processsus d'apprentissage et d'acculturation, eux mêmes tissés en partie de catégories linguistiques, dans l'apparente évidence des affordances ${ }^{8}$.

\subsection{La critique énactiviste}

17 Les chercheurs qui, autour de Varela, ont développé la théorie de l'énaction en dépassement assumé des cognitivismes classiques, rappellent de leur côté que la notion d'affordance renvoie aux "occasions d'interaction que fournissent les choses de l'environnement par rapport aux capacités sensori-motrices de l'animal» (Varela, Thompson, Rosch, 1993: 275). De fait, pour rendre compte de la perception, Gibson partage la dissolution de la représentation symbolique avec le néoconnexionnisme ${ }^{9}$. Cette démarche commune fait en effet l'économie d'une re-présentation du monde, directement perçu. L'approche écologique a d'ailleurs, en réponse à certaines critiques, précisé que la réciprocité animal-environnement portait fondamentalement la perception directe. Une réelle proximité conceptuelle permet du reste d'associer à cette dernière notion celle de couplage structurel entre le sujet (l'animal) et l'environnement, qui se trouve chez Varela et alii (1993 : 275-277) au cœur de l'énaction et de la saisie du monde médiée par des actions sensori-motrices.

En revanche, quant à la réciprocité elle-même, Varela souligne qu'elle n'implique logiquement pas la réponse à des invariants, au sens de l'acte de perception directe. Il concède que l'hypothèse repose sur l'expérience et ne peut être évaluée au plan strictement logique. Mais cette insistance de l'approche écologique de la perception reposant sur les invariants et intégralement assignée à l'environnement, délaisse l'autonomie de l'animal au prix d'une impasse sur la codétermination entre l'environnement et l'animal et surtout sa teneur (cf. Varela, Thompson, Rosch, 1993: 277-278). La mise au jour de la notion de couplage structurel, pour rendre compte de la congruence entre les clôtures du système nerveux et la signification qu'elles pourvoient, précisément, pour faire sens de l'environnement. Cette respécification théorique de l'invite, au sens de sa complémentarité avec son entour, permet assurément d'en préciser le fonctionnement : elle en fait un objet d'explication et non l'explication en elle-même, pour paraphraser Latour (2010: 17). Précisément, cette relation au discours, fût-il explicatif, permet d'introduire à la dimension proprement langagière des invites ou, si l'on préfère aux rapports qu'entretiennent affordance et langage. 


\section{Langage et affordance}

\subsection{Langage et forme du monde}

19 Pour résoudre le problème du vecteur sémiotique soulevé par Coulter et Sharrock, sans verser pour autant dans les pièges du logocentrisme, on convoquera les cadres théoriques de la linguistique anthropologique. Je souhaite prolonger ainsi les assomptions et le questionnement ouvert par Paveau (2013) dans une conception délibérément praxéologique. Partageant également l'intérêt l'auteure pour l'ancrage socio-historique, incorporé et du rapport au réel (qu'il s'agisse ou non d'artefacts), on rappellera, à sa suite, l'importance de ce faisceau de considérations pour rendre compte des fonctionnements discursifs et de la production de sens :

«les objets sont porteurs d'affordances linguistiques, c'est-à-dire de “linguistic possibilities", pour reprendre l'expression de Gibson. Ces affordances sont de différents types, qui restent à inventorier et à décrire: niveau du genre de discours, de la disposition graphique, de la forme prosodique, de la forme interactionnelle ou des différentes mémoires à l'œuvre dans le discours (mémoire sémantique, discursive). Ces affordances linguistiques sont permises par un certain nombre de traits de l'objet (son “design linguistique", en quelque sorte) concernant son rapport au langage » (Paveau, $2013: 55)$.

Qu'au-delà des objets, les faits, les événements soient, de même, vecteurs d'invites langagières, de potentialités linguistiques, ne constitue pas une extrapolation audacieuse : les systèmes sémiotiques du monde ambiant et de son continuum, au sein desquels les réflexions peirciennes nous ont accoutumés à distinguer indices, signaux et symboles font l'objet d'un travail interprétatif, pour le meilleur ou pour le pire. Très tôt, mythe et rationalité logique ont, du reste, œuvré ensemble, en miroir, à rendre compte de l'univers vécu. Les Anciens Grecs ne décelaient-ils pas indissolublement la signifiance des choses tant sous la rigueur mathématique ${ }^{10}$ que dans les Mystères d'Eleusis (cf. Eco, 2002 [1992] : 41 sqq) ? Aussi, si les données du réel, artificielles ou non, invitent à la catégorisation, à la dénomination ou se prêtent à l'indication et, plus ou moins à la glose ou la verbalisation, c'est bien en ce qu'elles pourvoient, littéralement, une relation à notre expérience comme êtres au monde. Pourtant, ce rapport - au sens à la fois saussurien et freudien où il n'y a que des rapports - revient à maitriser, par la tekhnê, d'abord, toute réalité expériencée dont l'utilité s'impose à nous comme son usage pertinent en tant qu'outil, prothèse de notre humaine condition. Pour redoubler de précaution contre le logocentrisme, rappelons que la tablette d'argile, façonnée à la main et pour la main a commencé par mémoriser, d'abord des données, pour ensuite, accessoirement, archiver des représentations verbales (réifiées ?). Le smartphone hérite à son tour de cette fonction haptique, qu'il nous invite en conséquence à activer... L'exemple est loin d'être isolé, dans le domaine des technosciences mais, plus généralement, le principe vaut pour l'ensemble des artefacts et peut même couvrir le mouvement inverse : ce sont les caractères chinois, eux-mêmes issus d'une longue culture de l'écrit et de l'iconicité, qui ont induit l'invention de l'imprimante laser, rendue nécessaire par leur finesse. Reste, quoi qu'il en soit, à déterminer la teneur du rapprochement entre langage et invite perceptuelle. 

l'environnement qui favorisent certains comportements sur d'autres »- au Lebenswelt de Husserl, Duranti précise que ce dernier

23 En l'occurrence, l'éclairage de l'anthropologie linguistique inspirée de la phénoménologie, permet de souligner que l'engagement dans les activités collectives du quotidien (comme, par exemple, la conduite automobile, guidée à la fois par la forme de la route et l'action des autres conducteurs) évite à chacun comme à tous d'avoir à discerner entre les savoirs et les pratiques. C'est sous cet aspect que l'on peut, me semble-t-il, intégrer pleinement la notation de Gibson rappelée supra à propos de la connaissance d'arrière-plan qui permet de savoir se servir d'une boîte aux lettres ${ }^{11}$. D'autre part, si l'on conserve la perspective de Duranti, la guidance des formes et des structures linguistiques, trouve à s'appliquer aux formats des genres discursifs comme à leurs horizons d'attente, à l'instar des cadres participatifs goffmaniens ${ }^{12}$... Peut-on, ainsi, déceler des affordances dans les données linguistiques elles-mêmes, c'est-à-dire au sens où les pratiques langagières, comme teknnê suscitent à leur tour des perceptions orientées vers la la réaction des sujets communicants, agissants? De même, un corps de sociolectes, de technolectes, ne peuvent-ils constituer des niches, au sens écologique, c'est-à-dire, selon Gibson (1979: 213), un « ensemble d'invites » qui impliquent un certain genre d'animal, auquel elles se conforment en retour, par distinction avec l'environnement phénoménal, le monde "privé » d'une espèce (ce que d'autres appellent Umwelt)? Mais cette reconsidération nécessite de différer encore l'application des affordances aux données linguistiques, si l'on souhaite en refonder conceptuellement l'ancrage écologique. En effet, les philosophies inspirées de l'Umwelt (ou «monde propre", que chaque agent perçoit, de façon individuelle ou collective, selon ses processus sémiotiques, pour vivre et agir en conséquence) du biologiste von Uexküll (1965 [1934]), appellent indirectement à repenser la notion même d'affordance dans un sens moins anthropocentré ${ }^{13}$ : que chaque organisme puisse habiter son environnement en relation avec sa réalité corporelle, ses aptitudes perceptuelles est une évidence. Dès lors TOUT devient a priori ressource disponible : seules celles qui entrent en adéquation et correspondance avec le corps et ses techniques (au sens de Mauss, $1966: 272^{14}$ ) de même qu'avec les développements cognitifs afférents, deviennent une réalité pertinente pour les praxis subjectives. Anthropologiquement, dans la longue chaîne historique et génétique qui relie ces fondements physiologiques et sociotechniques plus spécifiquement au langage, bien des mouvements évolutifs contribuent à déterminer, en autant de déterminations culturelles le rapport à l'environnement perçu. L'anthropologie linguistique a largement contribué à mettre au jour ces interrelations en termes relativistes, morphogénétiques et surtout non déterministes. Les êtres humains dans leur diversité individuelle et groupale partagent le même réel mais n'en retirent pas les mêmes pertinences : ce sont les différentes réalités (leurs facettes) que les langues mettent en spectacle. 


\subsection{Morphogenèse, signifiance et praxis}

La distinction entre affordances directes et indirectes, qu'elles nécessitent ou non un traitement différencié, pourrait bien être dissoute en continuum grâce à l'intéressant retournement que propose Simondon (2012[1958] : 327). Le déplacement théorique du philosophe consiste à dégager la part humaine consubstantielle aux objets techniques, et non seulement leur dépendance causale au travail (comme outil, aide, produit), ce qui suppose d'abord une inversion des rapports entre ce dernier et la technique.

"C'est le travail qui doit être connu comme phase de la technicité, non la technicité comme phase du travail, car c'est la technicité qui est l'ensemble dont le travail est une partie, et non l'inverse.»

En d'autres termes, la conception traditionnelle consiste à faire de la nature le pourvoi déterministe qui suscite le travail humain, adaptation de l'espèce pour surmonter ladite nature, en l'exploitant. Pour Simondon, le travail devient un cas de la technique, quand le porteur d'outils, en s'y prêtant, "réalise en lui-même la médiation entre l'espèce humaine et la nature » (Simondon, 2012[1958] : 328). Par le travail, l'homme opère donc une synergie entre matière (naturelle) et forme (intentionnelle), se donnant d'abord comme mutuellement hétérogènes. Si cette approche est contre-intuitive, c'est précisément parce que, comme le relève Simondon, la norme du travail incite le travailleur à se focaliser sur les deux termes précités, qu'il doit rapprocher, et non sur l'intériorisation de leur interaction (schéma hylémorphique). Les praxis, ici, sont un guide pour rendre compte de l'activité perceptuelle. La préparation du moule et le modelage de l'argile forment ensemble, le geste technique invité, que recèle encore l'outil.

On peut, à l'aune de cette conception des artefacts et du fonctionnement opératoire des objets techniques, envisager l'interaction entre environnement et agent humain comme synergétique, dans laquelle la perception agit comme tekhnê, dans la mesure où elle est acculturée. La confrontation milieu-organisme s'introjette de la sorte, s'intègre au sein de l'individu, sans avoir besoin de passer par la finalisation ou l'intentionnalité. Elle, est proprement, dans le corps du sujet, à l'inclusion de sa vie cognitive.

\subsection{De la perception aux praxis ou : comment transformer le réel en réalités?}

Assumant avec la praxématique (qui, pas plus que Gibson, ne revendique d'immanence du sens) et un certain nombre d'autres courants, la reversion des données expérientielles dans l'activité linguistique et par sa médiation, je pose la relation du langage au réel comme processus d'appréhension du monde: adossé à la perception sensible, le linguistique dit la part destinée à l'action et à l'interprétation. L'invite perceptuelle vaut ainsi pour disposition à agir (Morgagni, 2011) ou à interpréter. Précisément, pour Gibson (1979: 140),

« La théorie de l'affordance se départit radicalement des théories existantes de la valeur et du sens. Elle commence avec une nouvelle définition de ce que sont le sens et la valeur. La perception d'une affordance n'est pas un procès de perception d'un objet physique à valeur libre auquel le sens est quelque peu ajouté en un sens à propos duquel nul n'est apte à s'accorder. C'est un processus de perception d'un objet écologique à valeur riche. » 

montrer que, pour une partie significative de production du sens (comment faire sens du monde), la sémiose basique des systèmes linguistiques repose sur la motivation. Cette iconicité entre les choses et les mots, ne se transfère pas nécessairement à la forme de ces derniers mais aussi à leur fonctionnement diagrammatique. Le corps humain, si présent dans l'expression linguistique, en particulier sous la variation métaphorique fondant notre système conceptuel (Lakoff \& Johnson, 1987 : 67) a servi d'étalonnage à ce rapport au monde, à l'espace et au temps, comme le soutient avec force l'hypothèse localiste (Petitot, 1985) qui associe homologiquement les structures linguistiques et l'organisation de l'expérience ${ }^{17}$. Par exemple, on peut déceler sous les indices référentiels, pronominaux et adjectivaux le travail d'une telle morphogenèse :

\footnotetext{
Dans certaines langues, on trouve, au lieu de marqueurs spécialisés que leur figement rendrait opaques, une trace encore reconnaissable de la manière dont est traitée l'ipséité, - à savoir un groupe nominal constitué d'un référent possessif venant en détermination d'un centre nominal, lequel signifie, selon la langue, "tête ", « corps » ou « âme ». Ainsi, " lui-même » est rendu par «sa tête " ou « son corps » ou " son âme" : peul hoore-mum (têtesa), shilluk (Soudan) rí (son corps), basque nere-burua (ma-tête), vietnamien mình (corps), géorgien tavisi et mordve prä (tête). (Hagège, 1992 : 118)
}

31 Le réel $\mathrm{du}$ corps, ses parties, pourvoient ainsi naturellement au processus de grammaticalisation dès sa source langagière, du fait de leur rôle orienté (se prêtant à la métonymie). Toutefois, sur cette base universelle (chaque organisme perçoit le monde au crible de ses aptitudes physiologiques), toute langue déploie une appréhension particulière de l'environnement et $d u$ réel pour faire des réalités conformes aux perspectives de la communauté qui la parle. Pour devenir une réalité au yeux de cette dernière, la forme langagière doit s'inscrire en cohérence, selon une règle de convenance 
pratique, appuyée sur ce que Benveniste appelle le prédicat de réalité ${ }^{18}$, dans la logosphère propre à l'idiome ou au groupe linguistique et déclinée en arthrologie (découpage articulé, en cohérence, des niveaux linguistiques et sémiotiques). Ce processus embrasse ainsi perception et interprétation, dans la perspective induite par la mise en spectacle du monde du système linguistique. C. Hagège relate qu'il a assisté, au cours d'une enquête de terrain au Cameroun, à la création in vivo d'un mot par son informateur qui cherchait, au cours de la conversation, à désigner le magnétophone du chercheur, en mbum, sa langue. Le locuteur s'est servi, pour ce faire, du procédé de composition pour forger l'expression "calebasse à paroles» ( (á́w-Bè). L'auteur ajoute que, pour être consacré par la communauté (ce qui était attesté dès l'année suivante dans ce cas), un néologisme doit à la fois se conformer aux usages de formation lexicale dans la langue concernée et combler de façon adéquate le vide qui apparaît quand un concept cherche encore sa forme linguistique (Hagège, 1993 : 26). Ce rappel de la formation néologique renvoie à la forme linguistique du réel et de l'agir, pris dans le faisceau de la culture et des ressources environnementales, dans lesquelles s'expérience la perception. On le voit, loin de s'en tenir à une vision purement sémiotique et logocentrique, c'est bien sur l'expérience praxique que reposent «l'ordre de choses» benvenistien, la convenance pratique praxématique ${ }^{19}$, la morphogénèse du sens de l'hypothèse localiste ou le relativisme de l'anthropologie linguistique. Même si Gibson oublie de convoquer dûment la genèse des pratiques, la théorie de l'affordance, recoupe ces modèles à bien des égards. Partager un tel constat suppose que, d'une part, ni les contextes ni les environnements ne soient à considérer comme préconstruits, ou donnés et, d'autre part, que les visées des agents ne soient pas tenues pour finalisées. Du reste, bien qu'intuitive, la détermination par l'intentionnalité du comportement des organismes ne saurait être retenue comme une explication systématique : qu'on songe au processus de phagocytage des amibes, dont la causalité réside dans l'interaction entre les propriétés physico-chimiques d'enregistrement de la membrane et de mouvement du pseudopode (Foley, 1997 : 8-9). Rien, dans cette corrélation interne, qui soit conduit par un objectif à atteindre. Bien entendu, l'équipement neuronal des espèces douées de système nerveux déploie exponentiellement de telles aptitudes biologiques mais le principe demeure fondamentalement le même. Concernant les fonctionnements cérébraux eux-mêmes, Minsky, que je cite après Foley (1997: 9) - cf. aussi Lafont (1994: 325) - , a souligné combien « la principale activité des cerveaux consist[ait] à produire des modifications sur eux-mêmes » (Minsky, 1987: 288 - je traduis), dans la mesure où le tout repose sur des processus d'auto-changement ${ }^{20}$ : impossible, dès lors, de distinguer les processus des produits.

32 Si les faits (événements, objets, etc.) sont partagés, il faut envisager les situations comme des phénomènes vécus subjectivement. L'affordance correspondrait ainsi à un potentiel vectorisé : l'instance concernée oriente son usage par ses propriétés mais sur la base d'un fonctionnement indexical. En effet, l'indexicalité, propriété qui se rapproche le plus directement des processus de reconnaissance et de sémiose pertinente, en rapport avec les invites perçues, consiste à faire usage linguistique des caractéristiques du contexte ${ }^{21}$.

Or, une conception postdualiste conséquente - dont on partage l'assomption avec Paveau (2013) - induit a minima de discuter la partition humain / non humain et, par incidence, nature vs culture. Même sans tenir compte de l'évacuation de l'intentionnalité à proprement parler, la continuité entre les environnements tissés d'événements non finalisés des deux «règnes» l'emporte sur leur relative distinction: au constat des 
comportements symboliques animaliers, force est de se rendre en effet à l'idée qu'ils partagent l'aptitude humaine à interpréter des symboles, à activer des fonctionnements émergents, sur la base d'interactions improvisées. Qu'il y ait autonomie des ordres issus de la complexité technique et du langage, est tout aussi indéniable. Mais cette propriété anthropologique suffit-elle à opposer radicalement les tendances à l'œuvre dans les affordances de second niveau (fortement culturalisées et ritualisées) et celles qui relèvent des invites primaires? Tout se passe en effet comme si les caractéristiques émergentes et interactives étaient fondamentalement de même nature, hors spécificité anthropocentrée, ou comme, au contraire, si la dimension interprétative marquée dans les affordances complexes reposait sur le langage et l'activité technique (vectorisé par le schème hylémorphique simondonien).

\subsection{Catégorisation et pertinence}

En tout état de cause, la conception émergentiste et interactionnelle de l'affordance indirecte que je retiendrai ici - celle, pragmatique, inspirée de Norman - repose sur la mise en relation entre des propriétés intrinsèques à un objet, une instance, un phénomène et la perspective de l'acteur se trouvant mis en sa présence. Encore faut-il y associer des éléments inductifs de ce que l'on peut faire des propriétés perçues : la culture (technique) peut certes y suppléer mais un contre objet peut aussi s'avérer éclairant (un clou n'a pas de signifiance sans un marteau). Une suite de symboles n'est pas interprétable, a minima, sans son code. L'expérience de la chambre chinoise de Searle qui questionne le formalisme du fameux test de Türing, indique les limites de la simulation humaine de l'intelligence artificielle. En retour, elle montre que la capacité d'affordance perçue demeure, à ce jour, l'une des différences significatives entre humain et machine. Reste à déterminer si en formaliser le simulacre suffira un jour à en restituer les effets.

On doit, par ailleurs, relier à la propriété de surface, intuitive, des dispositifs technologiques et de leurs interfaces les processus de production qui esquissent et conditionnent l'adéquation de l'artefact à telle ou telle utilisation. La perspective des concepteurs passe par les multiples détours techniques (Latour, 2010): quel usager ordinaire pourrait en effet faire sens de chacun des composants électroniques qui composent tel smartphone? En revanche, cette intentionnalité initiale peut parfaitement être dissoute contextuellement par l'action d'agents qui ne l'interprètent pas de la sorte et qui détournent - sciemment ou non - ainsi l'usage de l'objet à des fins locales, selon ce qu'ils en perçoivent. Se servir d'une chaise pour accéder à une étagère en hauteur, voire pour exécuter un numéro de funambule (cas de détournement assumé et spectacularisé), et non pour s'y asseoir, par exemple.

Pour en revenir au détour technique, on peut y voir une réalité sociotechnique (comme le souligne Latour, approcher la société ne saurait se limiter aux données strictement sociales) mais surtout un cadre d'existence partagé, qui relève d'une apparence singulière mais qui recèle une stratification de ses formats de production, au sens goffmanien.

Inviter à une action, en favoriser l'émergence suffit-elle à la prédéterminer? Certainement dans tous les cas où, précisément l'affordance n'est pas perçu par l'agent. Ce qui renvoie au caractère intentionnel, dans le cas de l'artefact, non, bien entendu d'environnement naturel, produit en vue de telle ou telle activité et afin d'y pourvoir. Dans cette configuration, l'intentionnalité, qui renvoie à la destination même de l'objet technique, est en quelque sorte condensée dans le produit. Le jeu de telles propriétés 
constitutives d'affordances, orientées mais figées, renvoie à l'équivalent du travail mort des machines-outils selon l'économie marxiste, ou du savoir en dépôt que constituent les ressources linguistiques pour les ethnométhodologues. De l'expérience accumulée (le réel des sujets-agissants, leur praxis en dépôt, pour relire l'exemple de la boîte à lettres gibsonienne) et transformée en essence (la réalité de l'objet), une transmission s'établit, qui assure à la fois la pérennité des usages tout en servant d'assise possible à des déploiements ultérieurs, fût-ce dans une progression non linéaire, mais par détournement. En y décelant aussi du reste la base des détours techniques latouriens, on retrouvera la pleine dimension culturelle avancée par Norman. Dans ces cadres, toute affordance perçue est nécessairement interprétée et donc socialement partagée. Par conséquent, l'émergence perceptuelle elle-même repose sur le fondement distribué des ressources disponibles intégrées et l'activité interprétative des acteurs.

\section{Pistes pour une synthèse}

\subsection{Affordance et émergence}

Je retiendrai des précédentes critiques les propositions de couplage structurel énactiviste au lieu de la coordination animal-objet introuvable et l'inscription séquentielle du processus perceptuel des interactionnistes. La discussion ouverte par Norman donne toutefois elle aussi à réfléchir. Suivant la place que l'on accorde à la dimension culturelle et acquise des «affordances» indirectes, la densification contemporaine des environnements technologiques modifie-t-elle, infléchit-elle les observations écologiques précédemment déclinées, même revisitées? La section qui suit se concentre sur les rapports de l'affordance aux cours d'action à la fois associés au langage et instrumentés.

Je reviendrai d'abord sur la situation de l'habile conducteur décrite en prologue du présent travail. Dans ce cas, la surprise joue sur les attentes déjouées : le contexte partagé est saisi comme ressort (c'est justement parce que les rues et les immeubles sont entretenus dans ce musée à ciel ouvert qu'est la fameuse ville toscane, souvent sans trottoir, que la voie publique du centre historique est fréquentée par une foule dense, que la prudence s'impose d'autant plus aux automobilistes, tolérés); mais la pertinence émerge surtout de la maîtrise technique. Les musiciens parlent souvent des instruments qu'ils pratiquent comme prolongement de leur main. Ici, c'est la hauteur, la forme, l'orientation et le placement du rétroviseur qui font corps avec le conducteur italien, et, contre toute attente (en instrument synergique de l'effet de surprise évoqué), en font une ressource pertinente, en contre emploi absolu : c'est, en général, ce qu'il faut éviter qui devient le but recherché, de même qu'il est inopportun de bousculer quelqu'un mais que l'on peut faire mine de le faire pour provoquer une réaction et s'en amuser avec luielle, ensuite. Cet exemple de modalisation au sens de Goffman (1991 [1974]: 52 sqq.), parenthèse interactionnelle, s'appuie littéralement sur une invite perceptuelle, d'un acteur social qui maitre de la situation et de ses instruments de travail et peut l'interpréter en détournement. C'est précisément sur ce type de fluidité et d'intelligence situationnelle, mêlant interaction et comportements sociotechniques, adaptés pas à pas aux ressources et aux circonstances d'un environnement évolutif, que je souhaiterais insister dans les lignes ci-après. 


\subsection{Affordances langagières et praxiques en (inter)action : une étude de cas}

40 L'étude de cas suivante, permettra de mettre en regard les affordances liées à l'usage d'objets techniques et l'indexicalité dans un cours d'action partagé. L'extrait transcrit cidessous est prélevé d'un corpus enregistré dans le cadre d'une enquête menée au sein d'un CSU : centre de surveillance urbaine (cf. Bonu et alii, 2013)22. Le dispositif, qu'illustre la photo ci-dessous, repose sur l'interaction téléphonique entre un policier municipal (désormais : PM) et deux des opérateurs (OP1 et OP2) du CSU. Ces derniers, qui assurent la gestion et la manipulation des caméras à partir de leur poste de travail, partagent une partie des écrans avec leur interlocuteur.



Figure $n^{\circ} 4$ : Vue d'ensemble du mur d'écran des opérateurs du CSU

41 L'enjeu (dont l'extrait ne saisit qu'un point instantané du développement, au cours de la soirée de vidéosurveillance) consiste à localiser, quantifier et suivre un groupe alternatif, «le carnaval des gueux », susceptible de se greffer au défilé autorisé du carnaval antillais officiel (dit : « Karakwela » et réinterprété en « Caracoles » par OP1). 


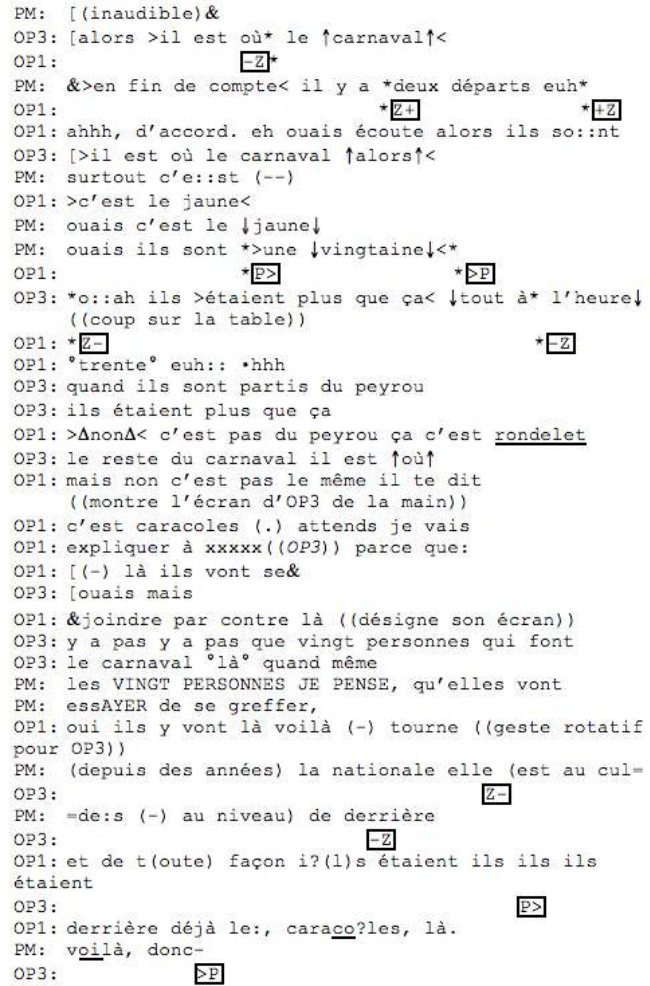

Figure $n^{\circ} 5$ : Transcription de la séquence

L'économie de moyens verbaux vient accompagner la réalisation praxique d'une tâche complexe qui passe par la distribution de l'interprétation des données vidéo en temps réel et sa traduction dans l'usage du dispositif sociotechnique qui sert précisément de cadre interprétatif.

L'environnement sociotechnique au sein duquel dialoguent et se meuvent les participants de cet échange, ne peut être considéré simplement comme leur cadre d'accueil, selon une perspective classique du contexte. Il s'agit d'abord d'un cadre participatif, au sens goffmanien. L'activité des interactants repose d'abord sur leur pratique usuelle et leur expérience, y compris partagée, en fonction de modalités un peu différentes, du reste, selon qu'il s'agit des relations professionnelles avec le policier et entre les deux opérateurs du centre. Mais si la coprésence de ces participants oriente en partie des actions engagées, les dispositifs technologiques s'associent à cette coconstruction, pour les guider partiellement. Les accomplissements sont ainsi à la fois partagés dans la mesure où ils sont contraints par les actions mutuelles et à travers l'éclatement des espaces (local de la police municipale, CSU, activités sur les écrans...) qui y contribuent également. Cette guidance distribuée des cours d'action, si complexe à décrire, et où les parts de chaque agent humain et celles de la technique s'entremêlent, procède pourtant, du point de vue de ses acteurs, d'une séquence d'action "naturelle», qui se déploie en quelque sorte pas à pas, selon des usages professionnels et interactionnels ordinaires. On soulignera d'abord que ces ajustements synchronisés progressivement n'ont rien de déterministe. Jusque dans la fusion des rôles participatifs (procédures de localisation, de quantification, de recherche, de désignation, de qualification, etc.), les accomplissements de chacun prennent en compte ceux d'autrui, comme les possibilités offertes par les objets présents. En ce sens, les objets techniques, les dispositifs, comme les humains et leurs actions (dont relève leur production langagière) sont mutuellement affordants ou, si 
l'on préfère, dans une conception cognitive distribuée, relèvent d'un étroit couplage structurel à effet coopératif.

Ce que met au jour par ailleurs leur séquentialité, c'est précisément l'évolution incrémentale des processus engagés, que représente le praxéogramme des cours d'action ci-dessous qui reprend synoptiquement une part des données transcrites divulguées supra . Les colonnes OP1 et OP2 indiquent les activités (notamment verbales) - à l'inclusion des gestes (en gras), faisant en particulier usage des caméras (surlignage) - des opérateurs face à celles du policier municipal (colonne PM); les quatre séries de cellules centrales synthétisent les principales verbalisations partagées, les flèches indiquant la directivité des adressages, d'une unité de construction de tour (UCT) à l'autre.

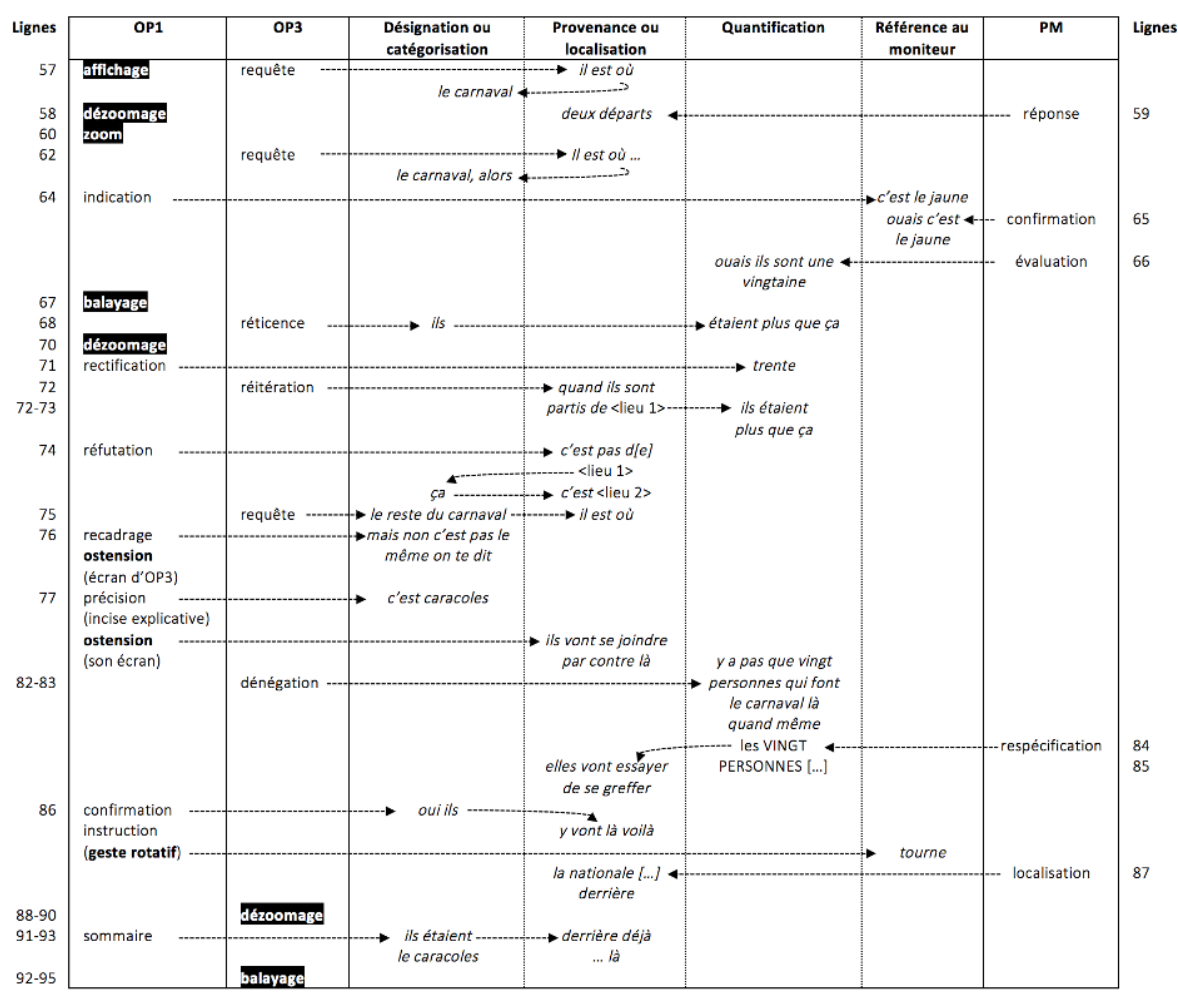

Figure $n^{\circ} 6$ : Table synoptique des cours d'action de la séquence

Le praxéogramme ci-dessus reproduit sommairement les principaux axes constitutifs de cours d'action de la séquence, autour des composantes qui forment les noyaux des UCT. Téléphone, claviers, souris, écrans informatiques et écrans vidéo partagés (à l'inclusion de leur lot d'étiquettes afférentes: code couleur des moniteurs, nom des rues où se trouvent les caméras, elles-mêmes numérotées...) pourvoient à la fois séparément et, plus exactement dans la séquence, ensemble, à diverses (inter)actions qu'elles permettent d'accomplir comme un tout supérieur en valeur d'effet à la somme de leurs réalités successives et distinctes (prises au sens unitaire). Il le font au-delà de ce pourquoi ils ont avant tout été ergonomiquement conçus. Gestes techniques, communicationnels ou symboliques s'associent de leur côté aux regards et aux énonciations verbales pour effectuer les tâches finalisées permettant de résoudre le problème professionnel considéré. L'ensemble sert notamment de support global à la cartographie en action à laquelle se livrent les participants et qui forme elle-même ressource pour les autres activités spécialisées à accomplir et précédemment déclinées... L'image mentale de l'espace puise dynamiquement sans doute dans leur expérience antérieure (connaissance 
de la ville, du quartier considéré...) mais repose surtout sur leur maittrise des dispositifs dont ils font le rhizome supportant leur improvisation, pourvoyant elle-même à l'interprétation partagée et à la bonne conjugaison des efforts conjoints pour répondre à la requête globale.

Pour focaliser sur un seul exemple, celui de l'extrait des lignes 84-86 de la transcription, on observe que l'opérateur, au téléphone, traduit gestuellement la quantification du groupe de carnavaliers estimée par le policier en indiquant à son collègue (occupant le poste de travail à sa droite) le mouvement à impulser à la caméra qu'il gère. Ce faisant, il confirme à la fois l'énoncé de PM et répond aux interrogations d'OP2 qui ne sait pas où chercher le groupe dans le défilé qui s'étire par les rues de la ville. Le mouvement rotatif de l'index, du poignet et de l'avant-bras de l'agent duplique de la sorte le... bras articulé de la caméra, en un geste générique et local de pointage et d'accomplissement technique. La ressource corporelle, doublée de la guidance verbale (voilà, tourne...) pourvoit naturellement, spontanément, à la description ou à l'explication, sans qu'OP2 ne manifeste la moindre surprise, ce qui assure de la valence partagée du geste signifiant.

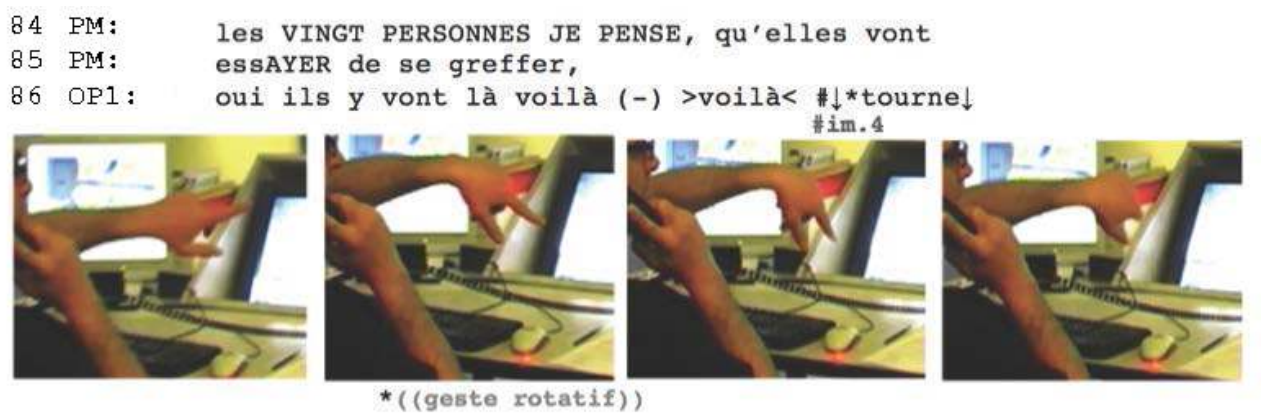

Figure $n^{\circ} 7$ : extrait de la séquence (rotation indicative)

Sur l'écran, les données perçues sont examinées à l'aune des attentes finalisées : quelles informations sur le groupe que l'on cherche à suivre sont susceptibles d'être pertinentes? Tout se passe comme si pour les opérateurs (et plus longuement pour OP1), le groupe était, sous ses diverses désignations et caractérisations (carnaval, ça c'est <lieu 2>, c'est pas le même, c'est Caracoles) - lesquelles, passent par la quantification (vingtaine, trente, plus que ça, que vingt personnes) - en soi, le carnaval, ce qui explique le différentiel entre leur nombre, assez réduit et le défilé imaginé (il est où le reste du carnaval ?). Cette conception évolue, non seulement, grâce aux précisions du policier municipal (deux départs, ouais ils sont une vingtaine, les vingt personnes elles vont essayer de se greffer) mais encore à l'aide des spécifications induites par l'usage de la vidéo, qu'il soit gestuellement (tourne + [geste rotatif] et / ou verbalement accompagné : c'est le jaune, ouais c'est le jaune... voire sans glose particulière : jeu des affichages, zoomages-dézoomages et autres balayages menés par les deux opérateurs. Interprétations progressives et perceptions graduelles se mêlent, inextricablement, dans l'abord des ressources et le cours d'action subséquent. Par opérations successives incrémentales, en autant de gestes professionnels, la technicité prend étai des corps praxiques et communicants comme de la maîtrise des dispositifs, dans un environnement fait de ressources et de potentialités émergentes. S'ils font corps avec ce pourvoi simultanément global et local, les agents sociaux considérés, sont tout autant en prise avec lui. Coconstruit dans les locaux des opérateurs et du policier, leur Umwelt s'étend à la fraction de l'espace urbain non seulement rendue visible par les caméras mais plus largement celle qui est rendue pertinente par le comportement ambulatoire du groupe visé, suivi, accompagné pour sa dangerosité potentielle dans 
l'espace public en tant que fauteur potentiel d'intranquillité et vu comme tel... Cette fin pratique fait sens tout en restant indissolublement en appui sur la perception progressive des données expérientielles et la forme, notamment linguistique, de l'interagir.

\section{Pistes conclusives : faire feu de tout bois ou les avatars du bricolage conceptuel}

«Il n'est rien qui naisse dans le corps pour que puissions nous en servir, mais ce qui naît engendre ensuite un usage »: déjà ces vers matérialistes de Lucrèce (De Rerum Natura ${ }^{23}$ ) contredisaient le célèbre « la fonction crée l'organe », axiome que l'on doit à Darwin pour synthétiser la pensée d'Aristote, en vue de sa réfutation moderne. De fait, les paradigmes biologiques de l'évolution ont su montrer que ni cette dernière, ni la nature ne relevaient de plans, de finalités, ou de projets intelligents : puisant dans les données existantes du réel, les organismes s'y adaptent en les bricolant et en stabilisant les aptitudes qui en découlent quand elles leur confère une prévalence vitale, comme espèce, jusqu'au stade d'optimisation évolutive suivant.

$\mathrm{Au}$ fond, toutes les tendances définitoires convergent vers une conception biologique du rapport entre organisme et environnement. Plus précise que des formes d'adaptation au milieu, les modifications de second degré (que Norman appelle culturelles) constituent des formes stabilisées de fonctionnements d'interaction entre organismes et contextes qui les introjettent, en quelque sorte, soit dans leur pratique, par une séquence gestuelle appropriée et éventuellement en s'équipant d'outils externes (le silex, le marteau, le marteau-piqueur, etc., sont des redéfinitions améliorées, du pourvoi que constitue le poing fermé) soit en les intégrant à leur physiologie: la musique use des ressources cérébrales du langage qui lui-même utilise l'audition dont le sens est d'abord voué à l'équilibre et à l'orientation spatiale. Au cœur même du langage, en lien permanent avec les formes d'activités et les ressources utilisées à toutes fins pratiques, se déploient ces formats d'exaptation, pour emprunter l'expression aux sciences de la vie (Gould \& Vrba, 1982). Il y a donc un risque de circularité à oblitérer cette donnée élémentaire du vivant, car elle permet de concevoir le fondement naturaliste du couplage structurel et de ses manifestations cognitives et linguistiques.

Les retombées de l'approche écologique gibsonienne, d'abord négligées ou sous-évaluées par les linguistes, constituent d'indéniables ressources pour la mise en cohérence de diverses perspectives analytiques, que Paveau (2013) décline comme approches TDI et auxquelles il faut adjoindre les démarches praxéologiques. Cette intégration suppose toutefois plusieurs ajustements théoriques.

51 Aller vers une conception dynamique...

52 La notion d'affordance est-elle bien utile? Reste-t-elle pertinente, si tant est qu'elle soit définie? De fait, si prise au large elle demeure séduisante, dès que l'on s'efforce d'en faire un usage précis, son opérativité tend à se délitter quelque peu: en deçà des leçons praxéologiques et phénoménologiques, plus ambitieuse que la valence gestaltiste mais moins interactive que la notion de couplage structurel, flirtant par concession avec une assise culturelle sans genèse ni processus, elle loupe historiquement les acquis plus étayés de la cognition située et distribuée. Précisément, si on considère l'usage superficiel de la définition parfois répandu, on serait tenté d'y voir une échappée commode soit d'une forme de constructivisme naturaliste (nos sens sont induits par les propriétés des réalités 
environnantes ${ }^{24}$ ) d'un retour piteux à un déterminisme conceptuel (les objets nous guident dans nos choix, autant que dans nos actions). Pourtant, même si elle a pu parfois osciller vers ces tendances, la pensée écologique de Gibson ne saurait s'y réduire. Pour les linguistes, elle s'avère, de plus, un vaccin contre les réifications du sens, ou les tentatives de ramener l'activité interprétative à son calcul. Cette double prophylaxie s'adjoint du reste d'une conception dynamique des contextes qui préfigure les paradigmes et ethnométhodologiques et émergentistes, à l'inclusion de l'anthropologie symétrique d'un Latour, comme l'a à juste titre relevé Paveau (2012: 56 sqq), qui intègre le principe dans sa propre «lingusitique symétrique ». Le couplage structurel de l'école énactiviste s'avère sans doute mieux étayé, les approches de l'incorporation plus précises, dans leur technicité quant au lien entre organisme et environnement; souhaitant renouveler l'approche anthropocentrée de la psychologie perceptuelle, Gibson a sans doute évité à tort de questionner le sujet praxique. Il n'en reste pas moins qu'ayant su faire œuvre utile, il a anticipé et préparé bien des questions contemporaines qui le replace à juste titre au cœur du débat scientifique contemporain : il appartient à une famille d'auteurs cultivant le paradigme de la dialectique internaliste-externaliste en termes d'interactions et s'ouvre délibérément au réel expériencé. Pour ces raisons, au moins, l'affordance gagne à être toujours objet d'investigation et de débats, y compris en sciences du langage qui en retireront une critique toujours vive et utile de la conception représentationnelle, certes intuitive mais dont l'établissement devient de plus en plus problématique.

\section{BIBLIOGRAPHIE}

Gazi A. \& Bonini T. (2018), “'Haptically Mediated' Radio Listening and its Commodification : The Remediation of Radio through Digital Mobile Devices", Journal of Radio \& Audio Media , $25: 1$, p. 109-125.

Bonu, B., Fauré, L., Broth M., Selimanovski C. (2013), « Explorer la ville un mardi gras : voir, montrer, dire le "carnaval des gueux" dans un centre de vidéosurveillance ", In D. Londei, S. Moirand, S. Reboul-Touré et L. Reggiani. Dire l'événement. Langage, mémoire, société, Paris, Presses Sorbonne Nouvelle, p. 49-59.

Col, G. et Victorri, B. (2007). « Comment formaliser en linguistique cognitive ? Opération de fenêtrage et calcul du sens temporel », Corela [En ligne], HS-6, mis en ligne le 01 novembre 2007, consulté le 01 octobre 2011, URL : http://corela.revues.org/1504

Coulter, J. (1999), “Discourse and Mind", Human Studies, vol. 22, n ${ }^{\text {os }} 2-4$.

Coulter, J. and Parsons, E.D. (1991[2002]), “The Praxiology of Perception : Visual Orientations and Practical Action", Inquiry, vol. 33, nº 3.

Coulter, J. and Sharrock, W. (1998), “On J. J. Gibson : A Response to our Commentators”, Theory and Psychology, vol. 8, $\mathrm{n}^{\circ} 2$.

Csordas, T. (1994). Embodiment and experience. Cambridge, UK : Cambridge University Press. 
Duranti, A. (2016), Anthropology of Intentions. Language in a World of Others. Cambridge University Press.

Eco, U. (2002[1992]), Interprétation et surinterprétation, Paris, P.U.F., Formes sémiotiques.

Gaver, W.W. (1996), “Affordances for Interaction: The Social Is Material for Design”, Ecological Psychology, 8(2), 111-129.

Gibson, J.J. (1986) [1979], The Ecological Approach to Visual Perception, Hillsdale, NJ: Lawrence Erlbaum Associates.

Gibson, J.J. (2014) [1979], Approche écologique de la perception visuelle. (Trad. fçse. O. Putois), Paris, Dehors.

Goffman, E. (1991) [1974]. Les cadres de l'expérience. Paris, Minuit.

Gould, S.J., Vrba, E. (1982), "Exaptation - a missing term in the science of form”, Paleobiology, vol. 8, p. 4-15.

Hagège, C. (1992), « Le système de l'anthropophore et ses aspects morpho-génétiques ", in M.-A. Morel et L. Danon-Boileau (éds.) La Deixis, Colloque en Sorbonne, 8-9 Juin 1990. Paris, PUF, p. 115-121.

Ifrah, G. (1994) [1981], Histoire universelle des chiffres. L'intelligence des hommes racontée par les nombres et le calcul. Tome 1, Paris, Robert Laffont, Bouquins, Seconde édition.

Lafont, R. (1978). Le travail et la langue. Paris, Flammarion.

Latour, B. (2010), Cogitamus: Six lettres sur les humanités scientifiques. Paris, La Découverte.

Lewin, K. (1969)[1936], Grundzüge der topologischen Psychologie. Posthume deutsche Ausgabe, hrsg. von R. Falk \& F. Winnefeld. Bern, Huber.

Mauss, M. (1966)[1935], « Techniques du corps », in : Sociologie et anthropologie, Paris, P.U.F., p. 363-386.

Merleau-Ponty, M. (1945). Phénoménologie de la perception. Paris, NRF, Gallimard.

Minsky, M. (1997)[1987], La société de l'esprit, [The Society of Mind], Paris, InterÉditions.

Morgagni, S. (2011), « Repenser la notion d'affordance dans ses dynamiques sémiotiques », Intellectica, 2011 (1), 55, p. 241-267.

Nadel, J. \& Potier, C. (2002), « Imiter et être imité dans le développement de l'intentionnalité », in: J. Nadel et J. Decety (dir.), Imiter pour découvrir l'humain. Psychologie, neurobiologie, robotique et philosophie de l'esprit. Paris, P.U.F., p. 83-104.

Norman, D. A. (1993), « Les artefacts cognitifs », in B. Conein, N. Dodier, L. Thevenot, Les objets dans l'action. De la maison au laboratoire, série « Raisons pratiques ", n 4, Paris, éd. de l'EHESS, p. 15-34.

Norman, D. A. (1999), “Affordance, Conventions, and Design”, Interactions 6(3), p. 38-43.

Norman, D. A. (2002) [1988], The Design of Everyday Things. New York : Basic Books.

Paveau, M.-A. (2012), « Ce que disent les objets. Sens, affordance, cognition », in Quelle place pour les objets dans les sciences du langage et sciences de la communication ?, Synergies Pays Riverains de la Baltique 9, p. 53-65, [en ligne : http://ressources-cla.univ-fcomte.fr/gerflint/Baltique9/ baltique9.html]

Petitot, J. (1985). Morphogenèse du Sens. Pour un Schématisme de la Structure. Presses Universitaires de France, Paris. 
Quéré, L. (1999), «Action située et perception du sens », in : M. de Fornel \& L. Quéré, La logique des situations. Nouveaux regards sur l'écologie des activités sociales, Paris, Éditions de l'École des Hautes Études en sciences sociales, p. 301-338.

Simondon, G. (1989), Du mode d'existence des objets techniques. Paris, Aubier.

Varela, F. J., Thompson, E. \& Rosch, E. (1993), L'inscription corporelle de l'esprit. Sciences Cognitives et expérience humaine. (Trad. fçse.), Paris, Seuil.

Von Uexküll, J. (1965[1934]), Mondes animaux et monde humain, suivi de : La théorie de la signification, (trad. fr.), Paris, Denoël.

Werner, H. (1948[1926]). Comparative psychology of mental development, (Rev. ed.). Chicago : Follett.

Wittgenstein, L. (1961[1953]). Tractatus logico-philosophicus suivi de : Investigations philosophiques.

(Trad. fçse P. Klossowski), Gallimard, Paris.

\section{ANNEXES}

Conventions de transcription

\begin{tabular}{|c|c|c|c|}
\hline\{\} & évènements simultanés & 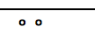 & volume plus bas \\
\hline [ ] & chevauchement de paroles & $<>$ & décélération du rythme \\
\hline (.) & micro pause ( $0.1 \mathrm{~s}$ ou moins) & $><$ & accélération du rythme \\
\hline$(n \cdot n)$ & pause minutée en secondes & MAJ & volume plus élevé \\
\hline & et dixièmes de secondes & inter- & segment inachevé \\
\hline$(--)$ & unité intonative interrompue & .hh & inspiration audible \\
\hline$=$ & continuité entre deux locuteurs & & (chaque " $h$ " correspondant à $0.1 \mathrm{~s}$ ) \\
\hline & (sans pause ni chevauchement) & : & allongement \\
\hline ? & montée élevée & * & repère du changement d'activité \\
\hline$\dot{c}$ & pic mélodique marqué & $P>P P$ & début et fin de panoramique vers la droite \\
\hline$\uparrow$ & mélodie montante sur la dernière syllabe & & en relation avec la parole en cours \\
\hline$\downarrow$ & mélodie descendante sur la dernière syllabe & $P<<<$ & début et fin de panoramique vers la gauche \\
\hline . & contour mélodique final descendant & $Z++Z$ & début et fin de zoom \\
\hline , & contour mélodique continuatif & $z-z$ & début et fin de dézoomage \\
\hline
\end{tabular}

\section{NOTES}

1. Sa conception renvoie à des objets-signaux qui nous poussent à agir. Voir, dans ce numéro, la contribution de C. Ruchon.

2. La topologie psychologique de cet auteur, cité par Gibson, entend sous l'affordance ( Aufforderungscharakter: "propriété d'invitation») la teneur d'une situation incitant ou déclenchant des actions, sachant que cette propriété n'est pas déterministe mais suscite une interaction de la situation (de terrain) et des besoins du sujet.

3. "The theory of affordances is a radical departure from existing theories of value and meaning. It begins with a new definition of what value and meaning are. The perceiving of an affordance is not a process of perceiving a value-free physical object to which meaning is somewhat added in a way no one has been able to agree upon; it is a process of perceiving a value-rich ecological object." (Gibson, 1979 : 140).

4. "Affordances provide strong clues to the operation of things. Plates are for pushing. Knobs are for turning. Slots are for inserting things into. Balls are for throwing or bouncing. When 
affordances are taken advantages of, the user knows what to do just by looking : no picture, label, or instruction is required."

5. «I believe that affordances result from the mental interpretation of things, based on our past knowledge and experience applied to our perception of the things about us. » (Norman, 2002 : 219).

6. Ces notations pourraient bien constituer le filigrane de la conception pragmatique de l'interjection selon Ducrot - grand lecteur de la philosophie analytique -, qui y voit une unité «arrachée au sujet». Le type de vocalisation interjective donnée dans l'anecdote liminaire du present article offre matière à discuter le rapport entre suprise du sujet et attentes sociales.

7. Pour une discussion plus détaillée des rapports entre Wittgenstein, la Gestalttheorie et la psyschologie écologique, cf. Quéré (1999 : 304-314).

8. On ne peut que rappeler, sans pouvoir y insister ici, les réflexions que Quéré (1999 : 312-336) a consacrées à la notion d'affordance. Son principal reproche porte plutôt sur le fait que la fonctionnalité de signification se distingue de la perception d'une signification dans une relation d'indication (laquelle suppose une normativité, celle des indices de Peirce). Autre distinguo: les affordances naturelles (primaires) sont d'une autre nature et nécessitent un autre traitement que les affordances secondaires socioculturelles.

9. La formule de mise en présence mentale, paraît plus adéquate que celle de représentation. Même si le concept même est discuté, on gagne à converger avec l'emprunt de la linguistique cognitive à la phénoménologie husserlienne qui lit en temporalité (nouvelle mise en présence discursive) et en déplacement attentionnel la construction du fenêtrage "représentationnel » en discours (Col \& Victorri, $2007: 2$ sq.).

10. Du reste, que les mathématiques soient, dans le droit fil de la conception pythagoricienne, le réel de l'ordre du monde mis au jour ou un système symbolique d'interprétation qui permet de rationaliser humainement les données de l'expérience, ne semble pas tranché. Ainsi, en dépit de la conviction de nombre d'auteurs, l'actualité des travaux précurseurs de Turing sur la morphogénèse participe de cette interrogation sur le déterminisme des lois de la nature et les rapports entre forme et fonction chez les organismes sans divulguer de réponse ferme quant à la nature du rôle des mathématiques.

11. C'est aussi ce qui fait la difficulté de la conception gibsonienne, trop universelle pour saisir réellement les spécificités culturelles. Mais cela vaut aussi pour la diversité des formes d'intelligence : l'expérience du monde par un enfant rencontrant des troubles autistiques, par exemple, montre que les invites du monde ne vont pas de soi, tant elles reposent sur des attentes sociales implicitées, que sa théorie de l'esprit n'appréhende pas forcément spontanément (Sur cet « autre intelligence, cf. Nadel \& Potier, 2002 : 83-104).

12. On relèvera au passage que la formulation définitoire qu'il retient de l'invite perceptuelle chez Gibson se laisse rapprocher étroitement de la notion de préférence en analyse de conversation, dont se réclame par ailleurs l'auteur.

13. À ceci près, toutefois, que selon ces auteurs, le monde sémiotique, chez l'homme, se distingue, par sa qualité d'expression du symbolique.

14. «Le corps est le premier et le plus naturel instrument de l'homme. Ou plus exactement, sans parler d'instrument, le premier et le plus naturel objet technique, et en même temps moyen technique, de l'homme c'est son corps ». L'exemple proposé en ouverture de ce travail me paraît valoir pour illustration de ce principe, appliqué précisément au véhicule comme prolongement du corps. V. aussi le développement donné, infra, en 4.1 et 4.2 .

15. « Le corps, rappelle Merleau-Ponty (1945: 97), est le véhicule de l'être au monde, et avoir un corps c'est pour un vivant se joindre à un milieu défini, se confondre avec certains projets et s'y engager continuellement $»$.

16. En leçon matérialiste du renversement des structures idéologiques, l'humanité conçoit ses langues à son image, dans sa diversité. 
17. Les conceptions anthropologiques de l'embodiment (Csordas, 1994), entre autres paradigmes, convergent avec cette approche.

18. Le linguiste en définit le concept ainsi : «le contenu de l'énoncé est (normalement) donné comme conforme à l'ordre des choses » (Benveniste, 1966[1950] : 154) .

19. S'entend par là la cohérence entre représentation discursive et réel représenté (Détrie et alii, $2017: 72-73)$.

20. Les récepteurs sensoriels se distribuent pour ce faire entre ceux qui réagissent à l'environnement et ceux qui réagissent aux changements internes.

21. Elle constitue, de fait, une forme généralisée de l'opération énonciative dite de deixis.

22. Les conventions de transcription sont données en annexe. Les toponymes sont sciemment banalisés en <lieu $1>$ et $<$ lieu $2>$.

23. Livre IV, v. 834-835 : «Nil ideo quoniam naturum est in corpore ut uti / Possemus, sed quod natum est id procreat usum. »

24. Ce qui, au passage est parfaitement en cohérence avec l'idée de mathématiques naturelles: l'ordre du monde est révélé par elles en lesquelles il tient.

\section{RÉSUMÉS}

Prise en un sens englobant (tel qu'initié par Werner, 1926), la notion d'affordance permet de penser en première approximation notre rapport perceptuel aux objets et surtout à ce que nous faisons du dit rapport comme sujets. Transférer cette notion psychologique à l'observation des données langagières (Paveau, 2013) paraît d'autant plus bienvenu qu'elle porte sur le rapport à l' activité de signifiance, au sens de Gibson (1979). Toutefois, outre les relectures successives qui ont contribué à en ajuster l'application à l'émergence (Turvey et alii, 1981) aux conventions culturelles (Norman, 1999, 2002), ou en tant que disposition à agir (Morgagni, 2011), certaines approches liées à la phénoménologie - ethnométhodologiques et énactivistes, en particulier - ont pu en discuter l'opérativité (Coulter \& Sharrock, 1998, Varela et alii, 1993).

Pour questionner sous sa robustesse la précision de ce concept tel qu'on peut en comprendre la manifestation en discours et en interaction, on posera le caractère transitoire de sa valeur opératoire avant d'observer comment il peut croiser diverses catégories praxéologiques (action, intentionnalité, ressources, indexicalité...). Une étude de cas d'utilisation d'artefacts pour visualiser et communiquer (dans un centre de vidéosurveillance) servira de support à la réflexion.

Taken in an all-encompassing sense (as initiated by Werner, 1926), the notion of affordance allows us to think as a first approximation our perceptual relation to objects and especially to what we make of this relation as subjects. Transferring this psychological concept to the observation of linguistic data (Paveau, 2013) seems particularly opportune as it concerns its relation to the significance activity, in the sense of Gibson (1979). However, in addition to the successive re-readings that helped to adjust its application to emergence (Turvey et al., 1981), to cultural conventions (Norman, 1999, 2002), or as a disposition to act (Morgagni, 2011), some approaches related to phenomenology -ethnomethodological and enactivist, in particular- have discussed its operativity (Coulter \& Sharrock, 1998, Varela et al., 1993).

To examine, under its robustness, the precision of this concept, as one can understand its manifestation in discourse and interaction, we set out the transitional character of its operative 
value. Then, we observe how it can cross various praxeological categories (action, intentionality, resources, indexicality ...). A case study which implicates using artifacts to visualize and communicate (in a CCTV center) is taken as a support for our investigation.

INDEX

Mots-clés : Praxéologie, perception, phénoménologie, énaction, indexicalité, signifiance.

Keywords : Praxeology, perception, phenomenology, enaction, indexicality, significance

\section{AUTEUR}

LAURENT FAURÉ

Université Paul-Valéry Montpellier 3 